\title{
Computed Analysis of Bone Density on Panoramic Radiographs Following Management of Benign Odontogenic Cystic Lesions
}

\author{
Amneet Sandhu ${ }^{1}$, Tejinder Kaur ${ }^{2}$, Ramandeep S Bhullar ${ }^{3}$, Sarika Kapila ${ }^{4}$, Amit Dhawan $^{5}$, Balwinder Singh ${ }^{6}$
}

\begin{abstract}
Introduction: Cystic lesions are one the most common pathologies in the oral and maxillofacial region. A number of software are available to assess the progress of bone healing following their surgical management.

Aim and objective: The aim and objective of this study was to assess the changes in size and bone density following treatment (either marsupialization or enucleation) of benign odontogenic cystic lesions in eight patients using computer analysis on pre- and postoperative panoramic radiographs. Materials and methods: The study was undertaken in 8 patients with mean age of 35.37 years (range $=11-50$ years). The male-female ratio was 3:5. All lesions $(n=7)$ were present in the mandible except one that involved right maxilla. Out of the total eight cystic lesions, six cystic lesions were treated by marsupialization, and two cystic lesions were treated by enucleation.

Results: There was a significant decrease in cystic volume postoperatively in all patients treated by marsupialization $(n=6)$ and also decrease in size of radiolucent (in all 8 patients) area radiographically, with progressive increase in bone density when compared to contralateral healthy side. The mean difference in bone density between preoperative and postoperative values was highly significant in the first 2 months, significant in third, fourth, and fifth month, and nonsignificant in the last 3 months. It was observed that the mean postoperative speed of shrinkage of the radiolucent areas was almost similar in the first 5 months and then decreased progressively over the next 4 months. The average speed of shrinkage was maximum for odontogenic keratocyst (OKC) $(n=3)$, followed by unicystic ameloblastoma (UAB) $(n=1)$, dentigerous cyst (DC) $(n=1)$, and was the least for residual cyst $(n=1)$.

Conclusion: The study noted that there might be a direct relation between size of primary radiolucent area and postoperative speed of shrinkage. Hence, digitalized orthopantomograph can be considered as an economical alternative to assess postoperative bone regeneration along with the added benefit of reduced radiation exposure to the patient.

Keywords: Benign odontogenic cystic lesions, Bone density, Panoramic radiographs.

AMEI's Current Trends in Diagnosis \& Treatment (2020): 10.5005/jp-journals-10055-0082
\end{abstract}

\section{InTRODUCTION}

The oral and maxillofacial region includes a variety of oral and paraoral structures from which a variety of pathologies such as cysts and tumors can originate. Odontogenic cystic lesion in the jaw is characterized as entities with benign biological behavior. ${ }^{1}$ Theses lesions have unique characteristics of being slow growing, but locally invasive and therefore may encroach upon adjacent vital structures and can reach considerable size if not diagnosed in time and treated appropriately. ${ }^{2}$ Although computed tomography (CT) scan is a more precise method to evaluate cystic lesions and postoperative bone regeneration over time, but it is too expensive, from both biological and economic view point, to be used routinely. ${ }^{3}$ These outcomes can be overcome by panoramic radiograph which is a relatively objective and useful method for evaluation of bone regeneration following surgical management of the cystic lesions. ${ }^{3,4}$ The present study was undertaken to determine the postoperative radiographic changes in the size of radiolucent area and also to determine the amount of bone regeneration following the treatment of various benign odontogenic cystic lesions using computed analysis of bone density on panoramic radiographs.

\section{Materials and Methods}

The sample for present study was selected from the patients reporting to the Department of Oral and Maxillofacial Surgery of our

\footnotetext{
1-60partment of Oral and Maxillofacial Surgery, Sri Guru Ram Das Institute of Dental Sciences and Research, Amritsar, Punjab, India

Corresponding Author: Tejinder Kaur, Department of Oral and Maxillofacial Surgery, Sri Guru Ram Das Institute of Dental Sciences and Research, Amritsar, Punjab, India, Phone: +919877793596, e-mail: tkgumber@gmail.com

How to cite this article: Sandhu A, Kaur T, Bhullar RS, et al. Computed Analysis of Bone Density on Panoramic Radiographs Following Management of Benign Odontogenic Cystic Lesions. AMEl's Curr Trends Diagn Treat 2020;4(1):1-10.

Source of support: Nil

Conflict of interest: None
}

Institute. Eight patients with benign odontogenic cystic lesions that could be subjected to enucleation/marsupialization were selected irrespective of gender, caste, creed, and socioeconomic status. Informed consent was obtained from each patient.

Patients belonging to American Society of Anesthesiologists (ASA) I (a normal healthy patient) and ASA II (a patient with mild systemic disease); patients with benign odontogenic cystic lesions which included odontogenic keratocyst, dentigerous cyst, radicular cyst, residual cyst, and unicystic ameloblastoma; or the patients who were compliant with the treatment protocol and could revisit for necessary follow-up were included in the study. On the other 
hand, patients with multiple recurrent lesions and patients in who the primary benign pathology had undergone secondary malignant transformation were excluded.

The diagnosis of the odontogenic cystic lesion was made based on detailed history, thorough clinical as well as radiological examination of each patient.

The radiographic evaluation of the lesion was done using digital panoramic radiographs and computerized tomography (CT) scan where indicated.

Prior to any surgical procedure, routine hematological investigations and complete urine analysis were carried out. Final diagnosis was confirmed from histopathological examination.

The preoperative assessment included evaluation of both clinical (type, location, volume, and size of cyst) and radiographic parameters (size and bone density). The volume was measured by injection of saline solution ( $\mathrm{NaCl} 0.9 \%$ ) into the cystic cavity at the time of marsupialization or enucleation after making surgical window and completely washing out cystic contents. Volume was measured 3 times, and the mean value was taken as its volume $(\mathrm{mL})$. The size was evaluated on panoramic radiographs. Standard lesion area was defined as: maximum vertical length $(\mathrm{mm}) \times$ maximum horizontal length $(\mathrm{mm})$.

Bone density was measured on panoramic radiograph using KODAK Dental Imaging Software $(6.12 .10 .0$ c Casestream Health, Inc. 2007, France). The measurement area was selected from central part of the cystic site and from healthy bone on the contralateral symmetric region of the panoramic radiograph.

The choice of treatment was either enucleation or marsupialization depending upon the size of cystic lesion, its location, and proximity to the vital structures. Marsupialization was the preferred method of treatment when the size of cystic lesion was large in lesions that were adjacent to important anatomic structures and also lesions involving unerupted teeth. The procedure was done under local anesthesia using intraoral approach in which a large surgical window was created in the cystic bony wall. After completely washing out the cystic fluid contents, the volume of cystic cavity was measured by injection of saline solution ( $\mathrm{NaCl} 0.9 \%$ ) into the cavity. This was followed by suturing the inner cystic lumen with the oral mucosa, thus interconnecting cystic cavity with the oral cavity. The cavity was packed with iodoform gauze after achieving complete hemostasis. The iodoform pack was changed at regular intervals till the lesion revealed clinical and radiological signs of decrease in size and formation of cortical bone in the areas of previous thinning which facilitated definitive surgical treatment. The total duration of marsupialization was noted. However, in large lesions, marsupialization was followed by enucleation. The time interval between both the stages was decided by clinical and radiological evidence of decrease in size of lesion and bone formation. Enucleation was generally advocated for small sized cystic lesions. It was also the treatment of choice if it could be performed without causing any damage to the adjacent structures. Enucleation with chemical cauterization using Carnoy's solution, and excision of the overlying mucosa was done in case of ododontogenic keratocysts. The histopathological examination of the pathological tissue was carried out. Postoperative assessment (on monthly basis) included the following parameters in cystic lesions that underwent marsupialization:

- Change in cystic volume

- Reduction in size of cystic cavity
- Speed of shrinkage = lesion area before marsupialization $\left(\mathrm{mm}^{2}\right)$ - lesion area after marsupialization $\left(\mathrm{mm}^{2}\right) /$ duration of marsupialization(months).

- Changes in bone density using panoramic radiograph.

The following parameters were evaluated postoperatively using panoramic radiograph in cystic lesion that underwent enucleation:

- Reduction in size of cavity.

- Change in bone density of affected area.

\section{RESULTS}

The mean age of the patients was 35.37 years (range varying from 11-50 years) with three male and five female patients, thereby making the male-female ratio to be 1:1.6 (Fig. 1). Figure 2 shows the type of lesions and distribution of each according to the site of occurrence in eight patients. Figure 3 shows the comparison between preoperative and postoperative volume of 6 cystic lesions treated by marsupialization. Preoperatively, the mean volume of these six cystic lesions that underwent marsupialization was 11.17 $\pm 4.58 \mathrm{~mL}$. The mean preoperative volume in one of the cyst that underwent enucleation was $2 \mathrm{~mL}$, whereas the other cyst treated by enucleation was located in maxilla; hence, volume could not be measured. It was observed that the cystic volume decreased on each postoperative visit.

Table 1 shows the postoperative change in volume of six cystic cavities treated by marsupialization when compared to the preoperative volume of the lesion. Preoperatively, the mean volume of cystic lesions was $11.17 \pm 4.58 \mathrm{~mL}$. Statistical analysis showed a statistically significant change in mean difference of volume postoperatively in all the patients $(n=6)$ when compared to preoperative cystic volume.

Figure 4 shows the comparison between preoperative and postoperative size of radiolucent areas, as determined on digital orthopantomograph.

Preoperatively, the mean size of eight cystic lesions treated either by marsupialization or by enucleation was $1191 \pm 742 \mathrm{~mm}^{2}$. The computed analysis of the postoperative radiographs showed that the mean postoperative size of radiolucent areas decreased with time irrespective of the mode of treatment (Table 2).

The statistical analysis showed that the postoperative reduction in size of radiolucent areas was significant ( $p$ value $<0.05$ ) in all patients $(n=8)$ when compared to the preoperative size on monthly postoperative follow-up. The complete shrinkage of radiolucent areas (lesions treated by marsupialization alone) was observed postoperatively on seventh month in residual cyst $(n=1)$ and at eighth month evaluation in OKC $(n=2)$. However, it was further observed that one case each of OKC, DC, and UAB which did not show complete shrinkage of radiolucent areas were enucleated in second stage procedure, after fifth month, eighth month, and ninth month postoperative period, respectively.

Out of two cystic lesions treated by enucleation, radiograph of the first patient showed complete shrinkage on fifth month evaluation and in second patient on sixth month postoperative follow-up.

Table 3 shows the speed of shrinkage of the cystic lesions treated by marsupialization as determined on digital orthopantomographs.

Preoperatively, the mean size of six cystic lesions treated by marsupialization was $1430 \pm 742 \mathrm{~mm}^{2}$. The computed analysis of the postoperative radiograph showed that the mean postoperative speed of shrinkage of the radiolucent areas with 


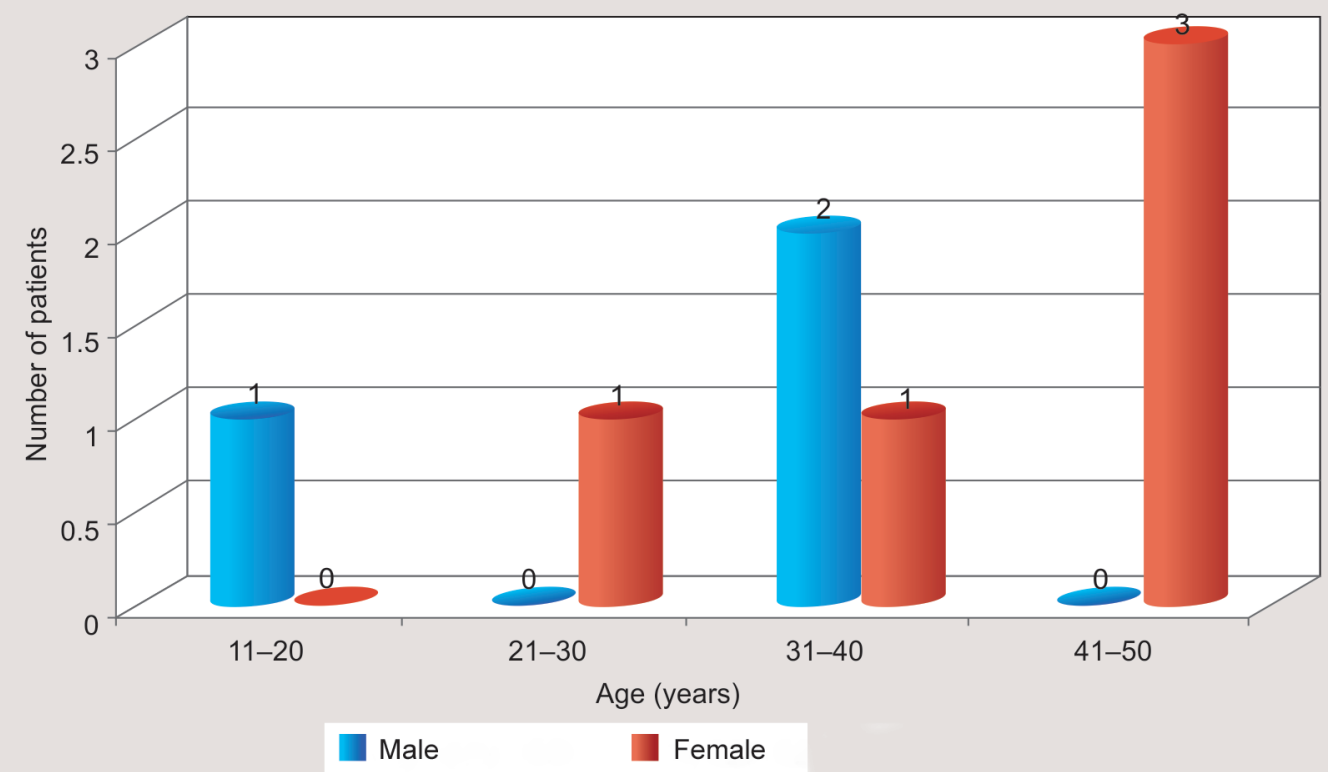

Fig. 1: Age and gender distribution

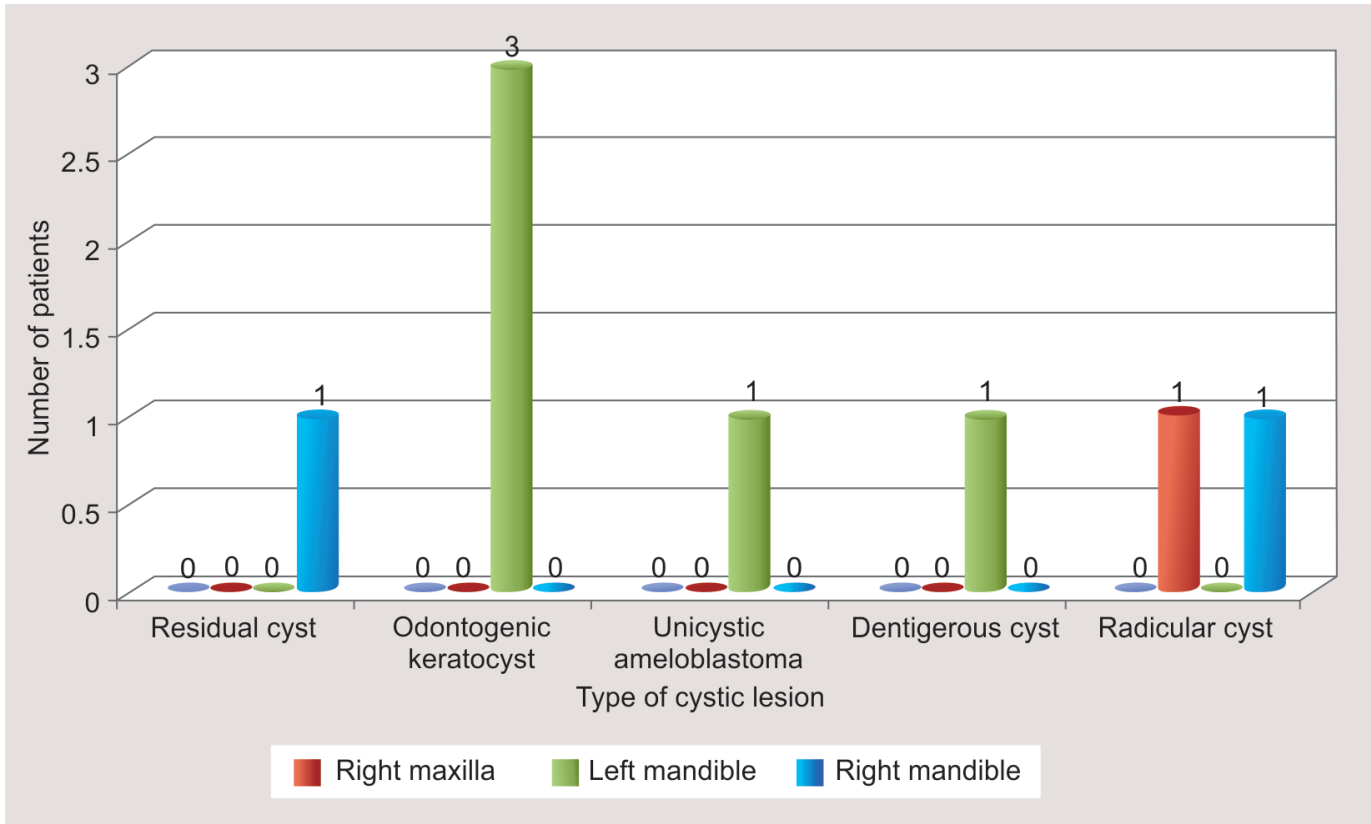

Fig. 2: Site and type wise distribution of lesion

time was almost similar in first 5 months (average $=278 \pm 163$ $\mathrm{mm}^{2} /$ month). It progressively reduced to $28 \pm 60 \mathrm{~mm}^{2} /$ month at ninth postoperative month. The mean speed of shrinkage for cysts treated by marsupialization was $797 \mathrm{~mm}^{2} /$ month for OKC $(n=3)$, $280 \mathrm{~mm}^{2} /$ month for $\operatorname{UAB}(n=1), 190 \mathrm{~mm}^{2} /$ month for DC $(n=1)$, and $129 \mathrm{~mm}^{2} /$ month for residual cyst $(n=1)$. Hence, the results showed that the speed of shrinkage was maximum for OKC, followed by unicystic ameloblastoma, dentigerous cyst, and was the least for residual cyst (Table 3 ).

Table 4 shows both the preoperative and postoperative bone densities of cystic cavities (affected side) when compared to the contralateral healthy side, as determined on digital orthopantomograph.

Preoperatively, the mean bone density of healthy and affected side was $125.38 \pm 9.15$ tonalities and $82.75 \pm 10.25$ tonalities, respectively. The computed analysis of the postoperative radiograph showed a progressive increase in bone densities of the cystic cavities on all postoperative visits (Fig. 5).

The statistical evaluation of difference in bone density of cystic cavity (affected side) when compared to the contralateral healthy side showed that the mean difference in bone density between preoperative and postoperative values was highly significant in 


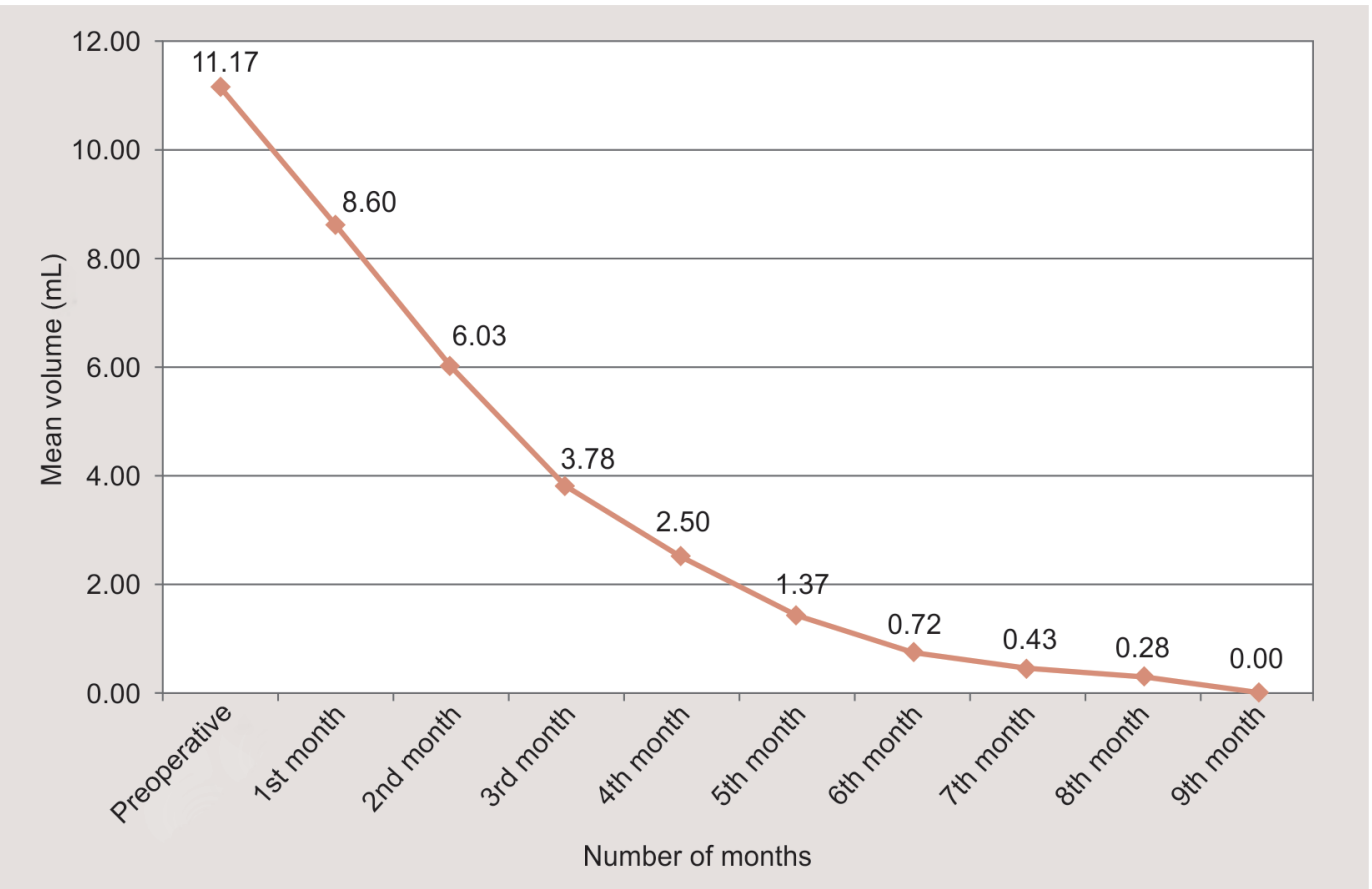

Fig. 3: Preoperative and postoperative volume of the cystic lesions

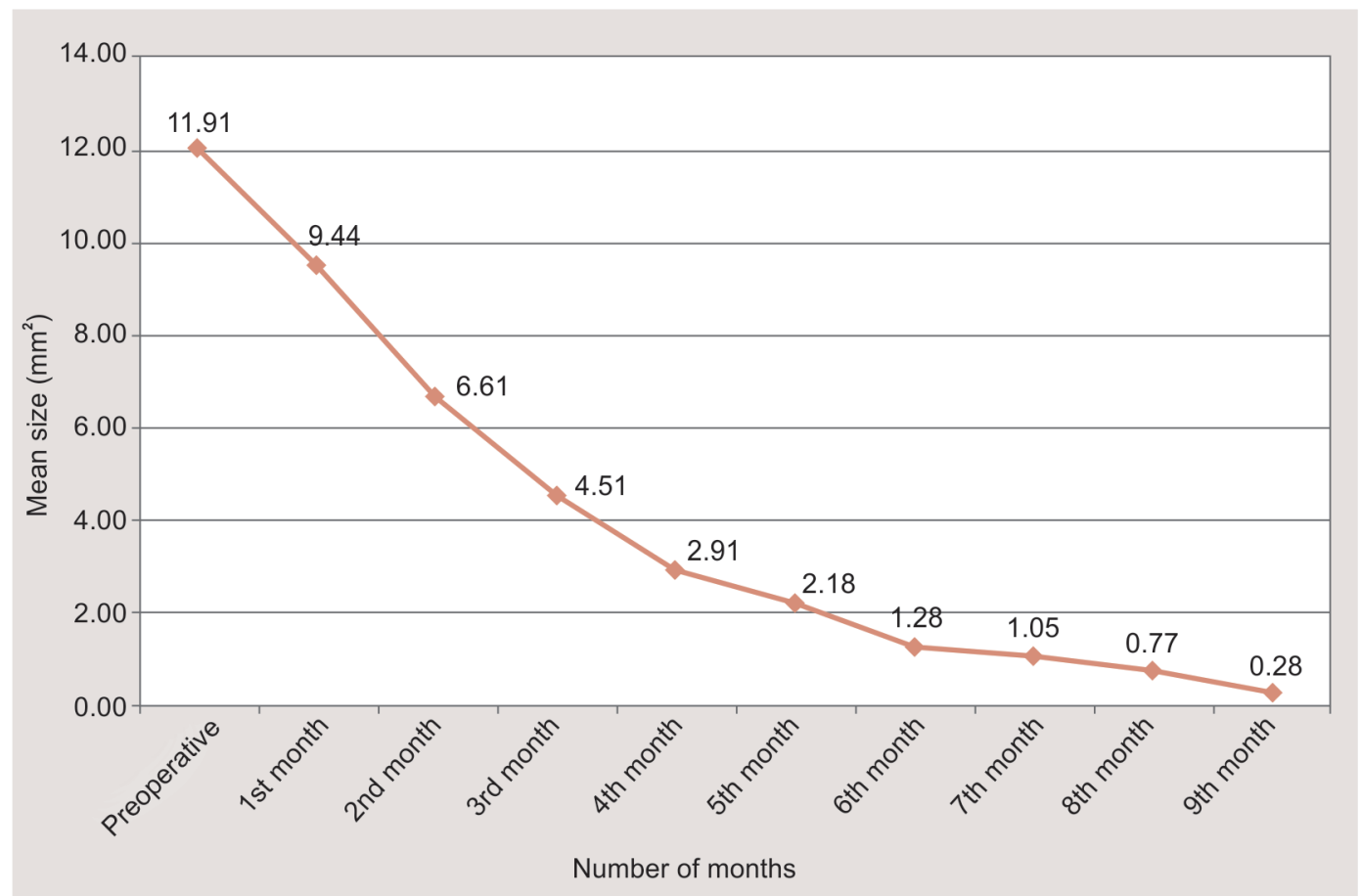

Fig. 4: Size of lesion on orthopantomogram

first 2 months, significant in third, fourth, and fifth month, and nonsignificant in the last 3 months (Table 5).

\section{Discussion}

Cystic lesions are one of the most common pathologies in the oral and maxillofacial region with great growth potential and can attain large size by resorbing the bone around the lesions. The capacity for bone regeneration in the jaw differs from that of other tissues, which mainly heal by forming scar tissue. This is mainly explained by the constant dynamics of bone resorption and apposition in normal bone. ${ }^{5-7}$

Radiography is the major nonoperative method for detecting bone formation in a healing osseous defect. Thus, it is more useful than histologic examination in clinical situations because of the speed, measurement continuity, and noninvasive nature. ${ }^{8,9}$ 

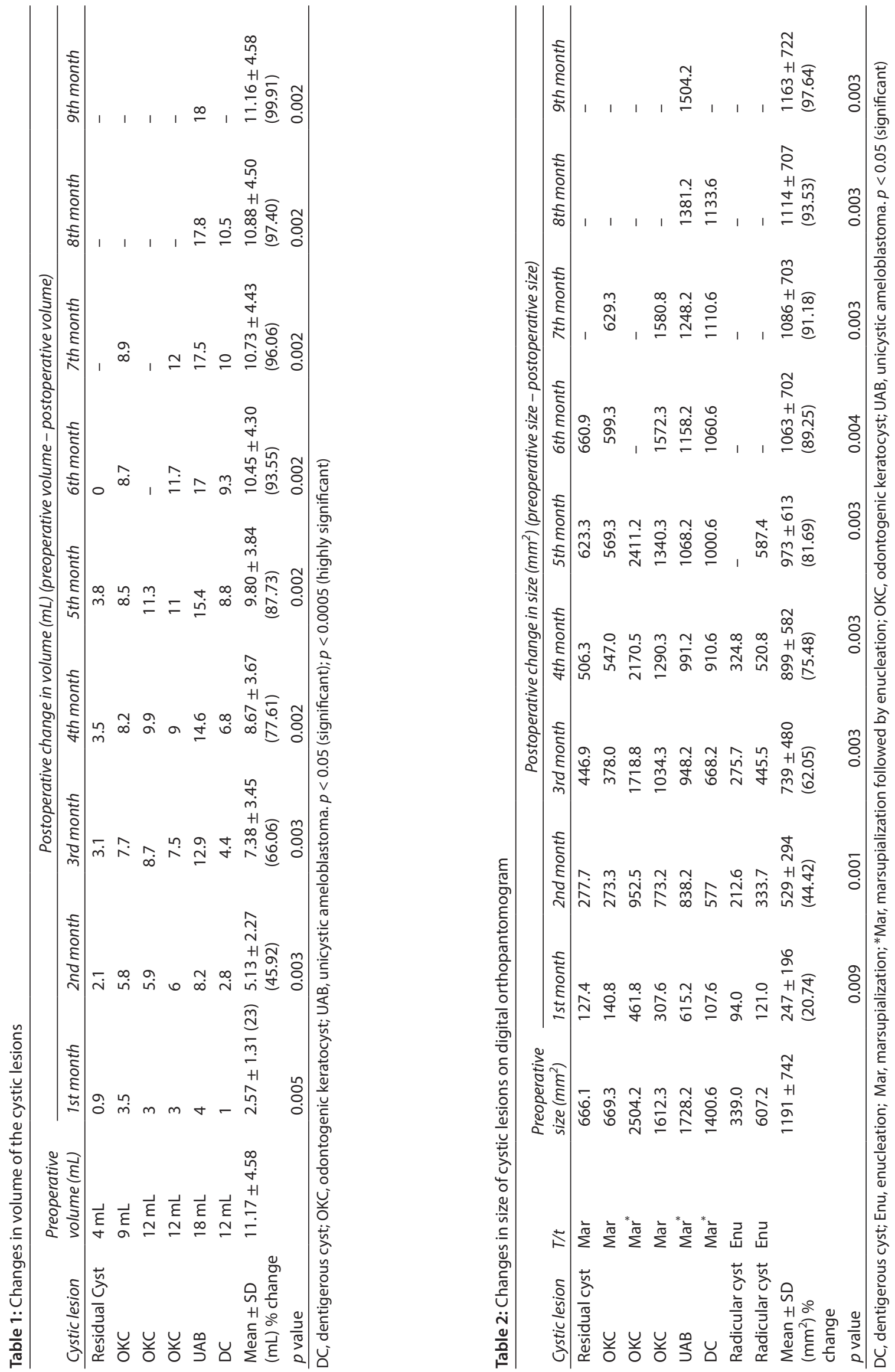


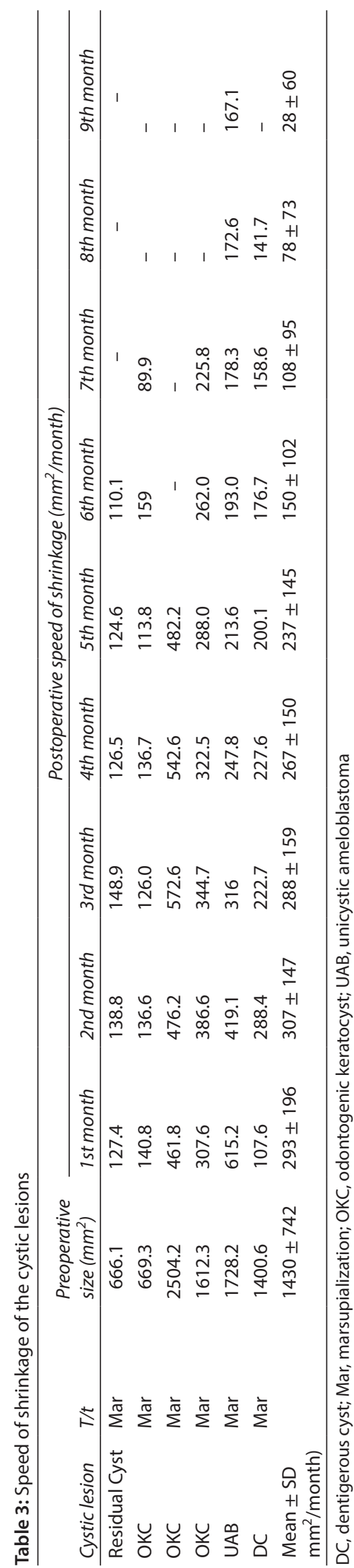

Digital panoramic imaging is a fast and excellent tool for evaluation of bony structures and for obtaining images of whole dentition in two-dimensional view. It gives overview of patient's oral health, facilitates communication with patients as well as other practitioners, better image quality, low economic costs, lower radiation hazards, and saves time, as there is no chemical processing. Bone formation is radiographically expressed as an increase in radiopacity, resulting in greater optical density of the bone image. To determine the progression of bone formation, radiopacity changes between radiographs should be compared using quantitative analysis and objective evaluation of the radiographic images. $3,10,11$

The present study was undertaken to determine the radiographic changes in size and bone density following the management of odontogenic cystic lesions. Age of the patients ranged from 14 to 46 years (mean age of 35.37 years). Out of eight patients, five (62.5\%) were female patients and three (37.5\%) were male patients making the male-female ratio to be 3:5. One lesion $(10 \%)$ was present in maxilla, while seven $(90 \%)$ lesions were present in mandible. Out of these seven lesions, three (42.85\%) were present in posterior region and four (57.14\%) lesions in the anterior region.

Preoperatively, the mean volume of the six cystic lesions treated by marsupialization was $11.17 \pm 4.58 \mathrm{~mL}$. It was observed that there was progressive decrease in volume of cystic cavity on every monthly postoperative follow-up. Statistical analysis showed significant change in the mean difference of volume postoperatively in all the patients $(n=6)$ when compared to the preoperative volume.

Asutay et al. ${ }^{12}$ also suggested that the ideal duration of decompression, depending on the size of the lesion, was when the volume decreased sufficiently for enucleation to be applied without damaging the vital structures. They also reported significant difference between preoperative and 6-month postoperative volume.

Preoperatively, the mean size of eight cystic lesions treated either by marsupialization or by enucleation was $1191 \pm 742 \mathrm{~mm}^{2}$ $\left(11.91 \pm 7.42 \mathrm{~cm}^{2}\right)$. Different studies in the literature have mentioned varying preoperative sizes $\left(2.60-25.35 \mathrm{~cm}^{2}\right)$ of the radiolucent lesions in jaws. ${ }^{5-7}$

The preoperative mean size of three cystic lesions treated by marsupialization alone was $666.1 \mathrm{~mm}^{2}\left(6.661 \mathrm{~cm}^{2}\right)$ for residual cyst $(n=1)$ and $1,140.8 \mathrm{~mm}^{2}\left(11.408 \mathrm{~cm}^{2}\right)$ for odontogenic keratocysts $(n=2)$. The complete shrinkage of radiolucent areas was observed postoperatively on seventh month in residual cyst $(n=1)$ and at eighth-month evaluation in odontogenic keratocysts $(n=2)$. Almost similar mean size $\left(14.5 \mathrm{~cm}^{2}\right)$ was reported by Anavi et al. ${ }^{13}$ in their study on decompression of 73 odontogenic cysts. The mean decompression time reported by the authors was 9.2 months. Similarly, Zhao et al. $^{3}$ also evaluated the bone formation after marsupialization of odontogenic keratocysts in 53 patients, with mean size of $5.4 \mathrm{~cm}^{2}$. The radiographic evaluation at 1 to 6 months after surgery showed gradual reduction of lesion size in all cases.

The preoperative mean size of 2 cystic lesions (both radicular cysts) treated by enucleation alone was $473.1 \mathrm{~mm}^{2}\left(4.731 \mathrm{~cm}^{2}\right)$. The radiograph of the first patient showed complete shrinkage on fifth month evaluation and in second patient on sixth-month postoperative follow-up. These results are quite similar to those of Chiapasco et al. ${ }^{14}$ who also evaluated the spontaneous bone healing after enucleation of large mandibular cysts in 29 patients with mean size more than $4 \mathrm{~cm}^{2}$. The clinical and radiographic evaluation 


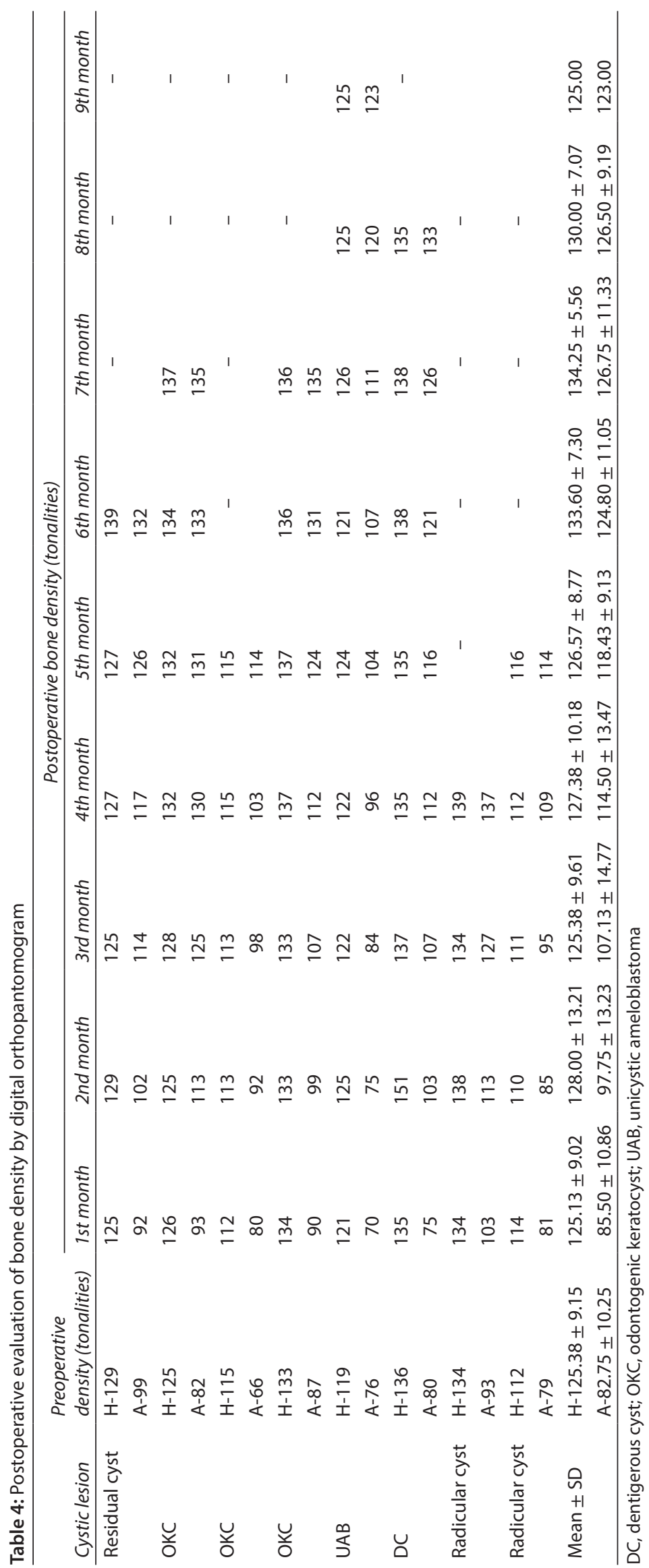




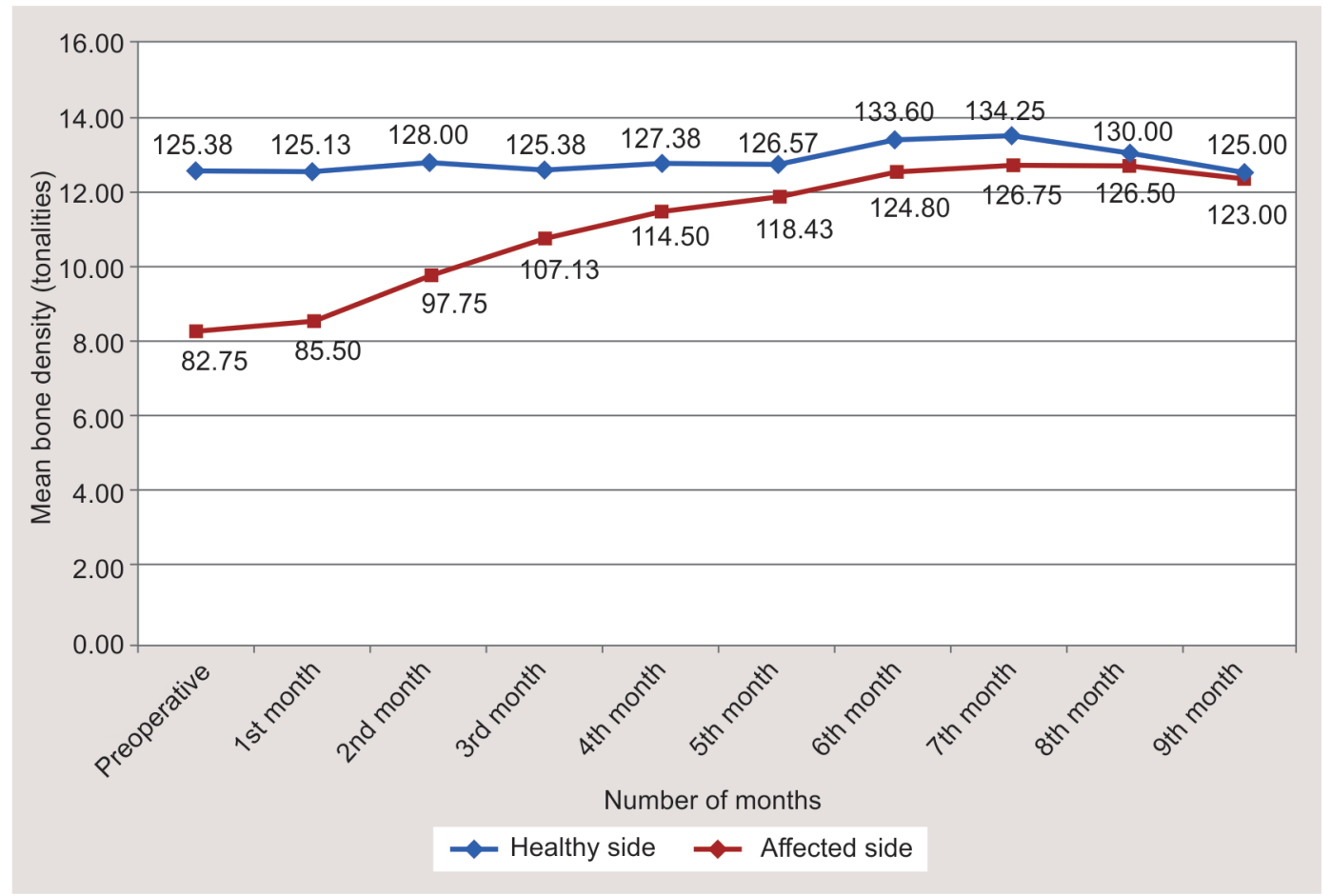

Fig. 5: Preoperative and postoperative evaluation of bone density by digital orthopantomogram

24 months after the surgical procedure showed complete healing of the lesions in all cases, with no sign of recurrence. Further, our results are supported by Ihan Hren and Miljavec ${ }^{15}$ who evaluated spontaneous bone healing of large bone defects in the mandible of 33 patients, with mean size more than $3 \mathrm{~cm}^{2}$. The authors observed a noticeable reduction in the size of bone cavity both clinically and radiographically during the first 6 months after enucleation.

Further, in our study, the mean preoperative size of 3 cystic lesions treated by marsupialization followed by enucleation was $2,504.2 \mathrm{~mm}^{2}\left(25.042 \mathrm{~cm}^{2}\right)$ for odontogenic keratocyst $(n=1)$, $1,728.2 \mathrm{~mm}^{2}\left(17.282 \mathrm{~cm}^{2}\right)$ for unicystic ameloblastoma $(n=1)$, and $1,400.6 \mathrm{~mm}^{2}\left(14.006 \mathrm{~cm}^{2}\right)$ for dentigerous cyst $(n=1)$. These cysts did not show complete shrinkage of radiolucent areas and were therefore enucleated in stage 2 procedure, after fifth month, ninth month, and eighth month evaluation, respectively. The results are well supported by Gao et al. ${ }^{2}$ who concluded that decompression tends to decrease the size of lesions and increased their bone density, but for aggressive lesions of jaw such as KCOTs, UABs, and DC, secondary definitive surgery is recommended. Similarly, Asutay et al. ${ }^{12}$ too suggested that decompression followed by enucleation might be a useful approach for the management of large cystic lesions.

The statistical analysis in our study showed that the postoperative reduction in size of radiolucent areas was significant ( $p$-value $<0.05)$ in all patients $(n=8)$ when compared to the preoperative size on every monthly postoperative follow-up. The computed analysis of the postoperative radiographs showed that the mean reduction of residual cavity to almost half (44.42\%) was attained by second month after marsupialization or enucleation, to three-fourth reduction (75.48\%) by fourth month, $89.25 \%$ by sixth month, and $97.64 \%$ by ninth month.

Our results are supported by Kubota et al. ${ }^{6}$ who concluded that the relative size of odontogenic cystic lesions treated by marsupialization, as indicated by the radiolucent area of lesions measured on panoramic radiographs, decreased in a linear fashion and in a time-dependent manner.

The computed analysis of the postoperative radiographs in the present study showed that the mean postoperative speed of shrinkage of the radiolucent areas with time was almost similar for the first 5 months (average $=278 \pm 163 \mathrm{~mm}^{2} /$ month) and then progressively reduced over next 4 months. The results showed that the speed of shrinkage was the fastest for odontogenic keratocysts followed by unicystic ameloblastoma, radicular cyst, dentigerous cyst, and the least for residual cyst. It was also noted that the speed of shrinkage of larger cystic lesions was faster than that of smaller lesions after marsupialization. These results are in concurrence with Gao et al. $^{2}$ who observed that the trends for relative shrinkage speed gradually decreased in KCOTs, RCs, and $U A B s$, respectively. The authors found a linear relation between the primary radiolucent area of cystic lesions before decompression and the relative shrinking speed after decompression among KCOT, $\mathrm{RC}$, and UABs, thereby indicating that the speed of shrinkage of larger cystic lesions may be faster than that of smaller lesions after decompression. The authors recommended decompression for all patients with large cysts, especially for older patients, to decrease surgical complications.

Similarly, Kubota et al. ${ }^{6}$ also reported linear reduction in the relative radiolucent area of the cystic lesions and concluded that the speed of shrinkage of larger cystic lesions might be faster than the smaller ones. They found that the mean relative speed of shrinkage of radicular cysts ( $11 \mathrm{~mm}^{2} /$ month) was significantly faster than that of KCOT or dentigerous cysts ( $7 \mathrm{~mm}^{2} /$ month).

Our results are also supported by Song et al. ${ }^{16}$ who found that there was a significant correlation between the initial volume of the lesion and the absolute speed of shrinkage in each type of cyst (keratocyst odontogenic tumor, unicystic ameloblastoma, and dentigerous cyst). 


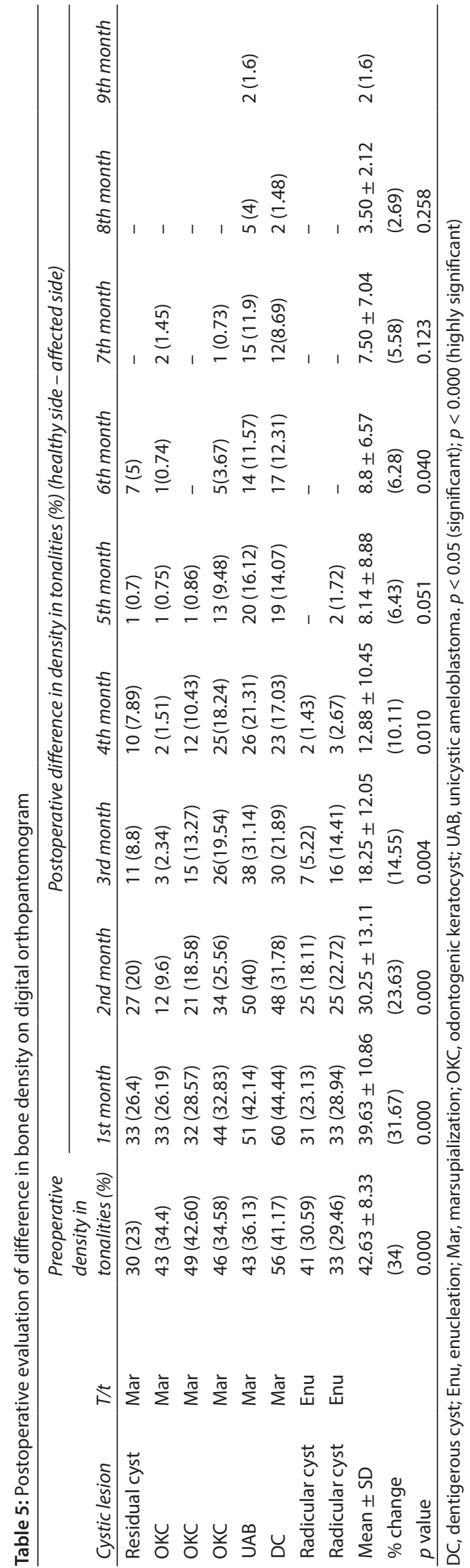

Bone density is the amount of bone tissue in a certain volume of bone. The preoperative mean bone density of healthy and affected side was 125.38 and 82.75 tonalities, respectively, thereby making the bone density of the radiolucent area to be $65.99 \%$. The computed analysis of the postoperative radiographs showed a progressive increase in bone density of the cystic cavities at all postoperative visits. It was observed that the mean bone density of radiolucent area was $68.32 \%$ at first month, $76.36 \%$ at second month, $85.44 \%$ at third month, $89.88 \%$ at fourth month, $93.56 \%$ at fifth month, $93.61 \%$ at sixth month, $94.41 \%$ at seventh month, $97.30 \%$ at eighth month, and $98.4 \%$ at ninth postoperative month, respectively.

Similar study was conducted by Gao et al. ${ }^{2}$ who showed that the increase in bone density of radicular cyst was the fastest (26.4\%), followed by odontogenic keratocysts (12.3\%) and unicystic ameloblastomas (9.0\%) as observed at regular follow-up of 1 to 24 months. Therefore, the authors hypothesized that the duration of decompression should be longer for aggressive cysts to increase bone density.

Our results are further supported by Gaikwad et al. ${ }^{17}$ who observed that the percentage increase in radiodensity of the cystic lesion ranged from 35.15 to $66.16 \%$. This indicated that the quantum of bone regeneration which occurred simultaneously and as observed during the cystectomy procedure was commendable.

Similar to the results of the present study, Chiapasco et al. ${ }^{14}$ also found that there was progressive increase in postoperative bone density of the radiolucent area. Compared to the immediate postoperative values, the mean increase in bone density was $37 \%$ at 6 months, $48.27 \%$ at 12 months, and $91.01 \%$ at 24 months postoperative follow-up.

The statistical analysis in our study showed that the mean difference in bone density between preoperative and postoperative values was highly significant in first 2 months, significant in third, fourth, and fifth month, and nonsignificant in the last 3 months. These results are in concurrence with those of Zhao et al. ${ }^{3}$ who also observed a progressive increase in the bone density of the cystic cavities at 3-month follow-up period. The mean increase in bone density of the cystic area was about $22.42 \%$ at 1 month, $46.07 \%$ at 3 months, and $64.69 \%$ at 6 months compared to the preoperative values. The increase in bone density was more significant in the first 3 months than in the next 3 months which is similar to the results of present study. They suggested that the significant increase in bone density and a remarkable decrease in the size of cystic cavity were found 3 months after marsupialization

The postoperative radiographs showed a progressive decrease in difference at all the postoperative follow-up periods which means that there was progressive increase in bone density at every follow-up. The mean difference in bone density was $31.67 \%$ at first month, $23.63 \%$ at second month, $14.55 \%$ at third month, $10.11 \%$ at fourth month, $6.43 \%$ at fifth month, $6.58 \%$ at sixth month, $5.58 \%$ at seventh month, $2.69 \%$ at eighth, and $1.6 \%$ at ninth postoperative month. Similar to the results of the present study, Zhao et al. ${ }^{3}$ suggested that the similarity in bone density between healthy and affected side following marsupialization of OKC in 53 patients was remarkable. Gaikwad et al. ${ }^{17}$ concluded that the change in radiodensity was indicative of two important things, first, the shrinkage of the cyst lining and second, simultaneous bone deposition along the walls of the cystic cavity, which was one of the major advantages of the decompression procedure. 
It is concluded from the present study that the ideal duration of marsupialization, depending on the size of the lesion, is when the size of the cystic lesion decreases sufficiently for enucleation to be applied without damaging the vital structures. The cysts that are smaller in size can be enucleated, whereas larger cystic lesion should be marsupialized, as it decreases the size of lesions and increases their bone density as evaluated on orthopantomogram. Hence, digitalized orthopantomograph can be considered as an economical alternative to assess postoperative bone regeneration along with the added benefit of reduced radiation exposure to the patient. The main limitations of the study are the small sample size and short follow-up time period of 9 months. Studies with larger sample size and longer duration of follow-up are required to reach definitive consequences.

\section{Informed Consent}

Yes.

\section{References}

1. Allon DM, Allon I, Anavi Y, et al. Decompression as a treatment of odontogenic cystic lesions in children. J Oral Maxillofac Surg 2015;73(4):649-654. DOI: 10.1016/j.joms.2014.10.024.

2. Gao L, Wang XL, Li SM, et al. Decompression as a treatment for odontogenic cystic lesions of the jaw. J Oral Maxillofac Surg 2014;72(2):327-333. DOI: 10.1016/j.joms.2013.07.035.

3. Zhao Y, Liu B, Han QB, et al. Changes in bone density and cyst volume after marsupialization of mandibular odontogenic keratocysts (keratocystic odontogenic tumors). J Oral Maxillofac Surg 2011;69(5):1361-1366. DOI: 10.1016/j.joms.2010.05.067.

4. Damante JH, da S, Guerra EN, et al. Spontaneous resolution of simple bone cysts. Dentomaxillofac Radiol 2002;31(3):182-186. DOI: 10.1038/ sj.dmfr.4600696.

5. Pogrel MA. Treatment of keratocysts: the case for decompression and marsupialization. J Oral Maxillofac Surg 2005;63(11):1667-1673. DOI: 10.1016/j.joms.2005.08.008.

6. Kubota Y, Imajo I, Itonaga R, et al. Effects of the patient's age and the size of the primary lesion on the speed of shrinkage after marsupialisation of keratocystic odontogenic tumours, dentigerous cysts, and radicular cysts. Br J Oral Maxillofac Surg 2013;51(4):358-362. DOI: 10.1016/j.bjoms.2012.07.017.
7. Ettl T, Gosau M, Sader R, et al. Jaw cysts - filling or no filling after enucleation? A review. J Craniomaxillofac Surg 2012;40(6):485-493. DOI: 10.1016/j.jcms.2011.07.023.

8. Crusoé-Rebello I, Oliveira C, Campos PS, et al. Assessment of computerized tomography density patterns of ameloblastomas and keratocystic odontogenic tumors. Oral Surg Oral Med Oral Pathol Oral Radiol Endod 2009;108(4):604-611. DOI: 10.1016/j.tripleo.2009.03.008.

9. Reddy MS, Mayfield-Donahoo T, Vanderven FJ, et al. A comparison of the diagnostic advantages of panoramic radiography and computed tomography scanning for placement of root form dental implants. Clin Oral Implants Res 1994;5(4):229-238. DOI: 10.1034/j.16000501.1994.050406.x.

10. Zhao Y, Liu B, Wang SP, et al. Computed densitometry of panoramic radiographs in evaluation of bone healing after enucleation of mandibular odontogenic keratocysts. Chin J Dent Res 2010;13(2): 123-126.

11. Apajalahti S, Hagström J, Lindqvist C, et al. Computerized tomography findings and recurrence of keratocystic odontogenic tumor of the mandible and maxillofacial region in a series of 46 patients. Oral Surg Oral Med Oral Pathol Oral Radiol Endod 2011;111(3):29-37. DOI: 10.1016/j.tripleo.2010.10.010.

12. Asutay $\mathrm{F}$, Atalay $\mathrm{Y}$, Turamanlar $\mathrm{O}$, et al. Three-dimensional volumetric assessment of the effect of decompression on large mandibular odontogenic cystic lesions. J Oral Maxillofac Surg 2016;74(6): 1159-1166. DOI: 10.1016/j.joms.2015.12.010.

13. Anavi $\mathrm{Y}, \mathrm{Gal} \mathrm{G}$, Miron $\mathrm{H}$, et al. Decompression of odontogenic cystic lesions: clinical long-term study of 73 cases. Oral Surg Oral Med Oral Pathol Oral Radiol Endod 2011;112(2):164-172. DOI: 10.1016/ j.tripleo.2010.09.069.

14. Chiapasco M, Rossi A, Motta JJ, et al. Spontaneous bone regeneration after enucleation of large mandibular cysts: a radiographic computed analysis of 27 consecutive cases. J Oral Maxillofac Surg 2000;58(9):942-948. DOI: 10.1053/joms.2000.8732.

15. Ihan Hren N, Miljavec M. Spontaneous bone healing of the large bone defects in the mandible. Int J Oral Maxillofac Surg 2008;37(12): 1111-1116. DOI: 10.1016/j.ijom.2008.07.008.

16. Song IS, Park HS, Seo BM, et al. Effect of decompression on cystic lesions of the mandible: 3 -dimensional volumetric analysis. Br J Oral Maxillofac Surg 2015;53(9):841-848. DOI: 10.1016/j.bjoms.2015.06.024.

17. Gaikwad R, Kumaraswamy SV, Keerthi R. Decompression and cystectomy of the odontogenic keratocysts of the mandible: a clinical study. J Maxillofac Oral Surg 2009;8(1):47-51. DOI: 10.1007/ s12663-009-0012-0. 